\title{
Las políticas culturales \\ en una nueva agenda de gobierno
}

\author{
Cultural policies in the government's new agenda
}

\section{Fernando Alonso Corbo*}

\footnotetext{
* Docente coordinador de la Unidad de Comunicación, Centro Universitario Regional Litoral Norte (Cenur), Universidad de la República, sede Salto.

$\bowtie$ fernandoalon@gmail.com.
}

\begin{abstract}
Resumen
La fuerza política al frente del Gobierno nacional estará culminando en breve su tercer período consecutivo en la conducción del país. En este lapso se han desarrollado un conjunto de políticas innovadoras a impulso del Gobierno, entre ellas varias en materia de políticas culturales. Desde 2005 el Uruguay ha procesado en diferente grado diversidad de cambios producto de las políticas implementadas. En ese orden, si bien varias metas se han cumplido, existe una nueva realidad que impone nuevos desafíos: ha cambiado el contexto social, cultural, económico y político, tanto interno como regional y mundial. Estos cambios interpelan al espectro político en su conjunto, pero principalmente al Gobierno y a la llamada agenda de gobierno. En el artículo se busca poner en discusión el rol de la cultura en esa futura agenda.
\end{abstract}

Palabras claves: política cultural, Uruguay.

\section{Abstract}

The ruling political party of Uruguay's national government will be shortly completing its third consecutive term in office. Throughout this period, innovative policies have been developed at the impulse of the national government, many of them referred to cultural issues. Between 2005 and the present time, Uruguay has processed, to a different extent, a diversity of changes as a result of the policies 
implemented. Although several goals have been met, a new reality imposes new challenges since the social, cultural, economic, and political context has changed, both nationally and regionally or globally. These changes question the political spectrum as a whole, but mainly the government, and challenge the so-called "government agenda". The article raises the issue of the role of culture in that future agenda.

Keywords: cultural policy, Uruguay.

\section{Introducción}

Desde el ascenso del Frente Amplio al Gobierno nacional se ha impulsado un conjunto de políticas culturales de gran volumen e impacto si se establece una comparación histórica. Sin embargo, en los últimos 13 años de gobierno no se han resuelto algunos de los problemas estructurales que permitirían terminar de arraigar el conjunto de acciones emprendidas y dar cumplimiento al programa de la fuerza política de gobierno, y principalmente a los acuerdos internacionales que Uruguay ha suscrito en cuanto a derechos culturales, ${ }^{1}$ referidos a garantizar mayor desarrollo, democratización y descentralización cultural.

Las principales políticas culturales impulsadas por el Frente Amplio, si bien han tenido continuidad, han sido desarrolladas con distinta intensidad en los tres períodos de gestión. También los gobiernos departamentales (en el presente artículo se toman tres casos conducidos por la misma fuerza política y con similares características) muestran criterios inverosímiles del rol de la cultura y las políticas culturales en la comuna.

Cuando se debate si el Gobierno nacional se ha quedado sin agenda o no, dos programas que aparecen como experiencias innovadoras en materia de políticas culturales públicas dejan al descubierto el potencial renovador que la cultura tiene para aportar en la agenda gubernamental. A ello se dedica el presente artículo.

Al llegar al gobierno nacional, el Frente Amplio comenzó una serie de innovaciones en materia de políticas culturales, procurando sacar al Uruguay del profundo retraso que presentaba en este campo y ponerlo en sintonía internacional. Se crearon por ley diferentes fondos de carácter concursable para estimular y fomentar la cultura,

1 La idea de ciudadanía cultural parte de principios y de una serie de convenciones y resoluciones que la UNESCO ha divulgado al menos desde 1998, con la Conferencia Intergubernamental sobre Políticas Culturales para el Desarrollo, celebrada en Estocolmo (UNESCO, 1998), en 2001 con la Declaración Universal sobre la Diversidad Cultural (UNESCO, 2004) y en 2005 con la Convención sobre la Protección y la Promoción de la Diversidad de las Expresiones Culturales (UNESCO, 2005), ambas aprobadas en París, todo en estrecha relación con la defensa de los derechos culturales y de la diversidad cultural. 
como el Fondo Nacional Concursable para la Cultura y sus derivados, los Fondos Regionales de Cultura, el Fondo para el Desarrollo de Infraestructuras Culturales en el interior del país, el Fondo de Incentivo, el Fondo de Estímulo a la Creación y Formación Artística y el Fondo de Fomento Cinematográfico y Audiovisual, se mejoraron fondos ya existentes y se impulsó la integración internacional a los denominados fondos Iber. También se crearon el Instituto del Cine y el Audiovisual, el Instituto Nacional de Artes Escénicas, el Sistema Nacional de Museos y el Sistema Nacional de Bibliotecas Públicas, entre otras vías de apoyo o estímulo directo.

A ello se agregan otras políticas, como la creación de los Centros MEC, las Usinas Culturales y las Fábricas de Cultura, que apuntan a saldar la histórica deuda de presencia física del MEC en todo el territorio nacional, principalmente el interior.

Sin embargo, el acelerado ritmo que tuvo esa agenda de políticas culturales —en general sin acompañamiento de una institucionalidad y una estructura gubernamental acordes - paulatinamente fue decreciendo. Una asociación directa puede relacionarla con la desaceleración del crecimiento del país desde principios de la presente década, aunque la desaceleración de las políticas culturales fue aún mayor que la economía del país. Una segunda causa seguramente está vinculada con la prioridad que adquirieron en la distribución presupuestal otras áreas del Estado, como seguridad, salud o educación. En el último tramo hubo problemas para consolidar muchas de las acciones encaminadas.

La interrogante que se plantea es qué sucederá con estas innovaciones -tomémoslas como logros - con un eventual cambio de orientación política en el gobierno nacional.

\section{Políticas de Estado}

En materia de políticas culturales, en comparación tanto con la historia uruguaya reciente - tomemos las últimas cuatro décadas - como con las experiencias regionales más próximas, se advierte una sustancial diferencia entre la perspectiva de los gobiernos de izquierda y centro-izquierda, por un lado, y los que podríamos identificar dentro del espectro liberal o de centro-derecha y derecha. Mientras los primeros le han adjudicado al Estado un rol preponderante en el impulso de políticas culturales, en especial las que despliegan líneas de democratización cultural, los segundos se han inclinado por un repliegue del Estado en este campo. La mentada baja del costo del Estado incluye reducir el presupuesto de cultura. También es notoria la ausencia de la temática cultural en las agendas partidarias de esta segunda perspectiva. 
Así como al inicio del presente artículo se listaron algunos avances en políticas culturales de los sucesivos gobiernos frenteamplistas, en el sentido inverso, de desconstrucción política, se pueden mencionar varias medidas que se han tomado en la República Argentina con la administración Mauricio Macri, desde los casos de mayor escándalo, como el despido de unos 600 trabajadores del Centro Cultural Kirchner en su primer año de gestión, hasta el más reciente cierre del Ballet Nacional, o la afectación de un sinnúmero de espacios culturales independientes y comunitarios diseminados por todo el país. Incluso la creación de una ley de mecenazgo llamada Sistema Nacional de Desarrollo Cultural, que podría tomarse como un posible avance, despertó fuertes polémicas en diversos ámbitos y colectivos de artistas que consideraron que pone el foco en el beneficio empresarial y no en el desarrollo de las artes.

Voces muy calificadas han aludido de modo directo y duro a este proceso. Néstor García Canclini habló de «la disolución del campo cultural» y afirmó que «en Argentina y en Brasil, gravemente, hay una reducción de los presupuestos científicos y culturales» (Mendoza, 2018).

Por su parte, Rubén Szuchmacher, actor, director y gestor teatral, reflexionó: «La facilidad con la que Macri desmonta hoy las políticas culturales del período anterior responde a la poca solidez que estas tuvieron» (Yang y Abraham, 2016).

\section{Asincrónico}

Los casos mencionados no se presentan como absolutos, sino como reflejos contundentes de una compleja realidad. Tomando la reflexión de Szuchmacher, el Uruguay tiene mucho para avanzar aún en las políticas y la gobernanza cultural, principalmente en situar a la cultura en su agenda de políticas de Estado.

Así como se aludió en grandes líneas al proceso que se está registrando en el Gobierno nacional, se puede traer el ejemplo concreto de tres gobiernos departamentales frenteamplistas que comparten una misma región: Río Negro, Paysandú y Salto.

En Río Negro, dentro del organigrama del gobierno departamental, la Dirección de Cultura (y Educación) está inserta en la Dirección General de Promoción y Desarrollo Humano. En Paysandú, la Dirección de Cultura tampoco tiene rango de dirección general, sino que se inscribe en el Departamento de Promoción y Desarrollo. En Salto, en cambio, Cultura sí es un departamento autónomo. ${ }^{2}$ La diferencia de partida está en que

2 En la Intendencia de Salto el Departamento de Cultura fue creado dentro del organigrama departamental en el período 2005-2010, del intendente Ramón Fonticiella, y continuó en el período 2010-2015, del intendente Germán Coutinho, así como en el actual gobierno del intendente Andrés Lima. Vale recordar que el presupuesto quinquenal del ejecutivo de Lima, si bien no fue aprobado por la Junta 
por tener esa jerarquía integra el gabinete departamental de gobierno, lo que marca una diferencia nada menor.

Más allá de la ingeniería aplicada, la impronta del accionar y la disponibilidad presupuestal son considerablemente diversas en los tres casos. Pero lo que interesa aquí es notar cómo tres gobiernos frenteamplistas, dos de ellos del mismo sector interno, tienen valoraciones distintas del rol de las políticas culturales, lo que se expresa en sus respectivos organigramas departamentales, con independencia de que en los hechos su accionar tenga más puntos en común que diferencias.

Estas perspectivas recorren un amplio espectro, entre concebir a Cultura como un área vinculada a Turismo y Deporte, como una de las políticas de desarrollo humano y social, e incluso relacionarla con el desarrollo productivo y económico, la innovación o la educación.

Esta región que se tomó como ejemplo dispone de un valioso repertorio de servicios culturales: teatros, museos, espacios expositivos, casas de autor y centros culturales, bibliotecas, anfiteatros y sitios patrimoniales. Es un enclave territorial de frontera estratégico y una de las regiones con mayor atracción turística nacional, así como el mayor polo de oferta de enseñanza terciaria y universitaria en el interior, lo que genera una amplia circulación de públicos.

No obstante, el ritmo de crecimiento de este enclave litoraleño está notoriamente por debajo del que tiene la región sur, y más aun del que esta proyecta. Hay una relación asimétrica que se irá acentuando, donde al río Negro le cabe más que nunca la metáfora de grieta norte-sur.

Del lado de los que piensan que la cultura puede actuar como una palanca de desarrollo integral, o al menos que no puede quedar al margen en una estrategia de desarrollo regional, ¿es posible concebir, dentro de un marco de institucionalidad, la ausencia de un enclave físico de gobernanza cultural del gobierno nacional en esta región, así como la ausencia de un espacio de coordinación regional de los gobiernos locales?

Como señalaban Pascual i Ruiz y Dragojević, «Implementar nuevas políticas culturales para la ciudadanía necesita una innovación institucional; en otras palabras, una nueva arquitectura de la política» (2007, p. 35). 


\section{Una agenda a renovar}

Como se expresaba al principio, actores de la oposición hablan de un agotamiento de la agenda política del Gobierno nacional, a lo cual se suman algunos analistas políticos que con otro tono coinciden en la necesidad de actualizar dicha agenda.

Creemos que en esa nueva o renovada agenda de gobierno la cultura tiene un papel clave. En parte porque la mejora en promedio del ingreso de los uruguayos, el aumento de su capacidad de consumo y el exponencial crecimiento del flujo turístico hacen un terreno fértil para el desenvolvimiento de un conjunto de acciones culturales. Pero también porque el desarrollo cultural por sí mismo promueve el fortalecimiento del entramado social y los procesos de inclusión, mejora las prácticas de convivencia y, sobre todo, eleva la calidad de vida de los ciudadanos.

No faltan propuestas innovadoras en este escenario de realidad interpelada, solo que en algunos casos vienen avanzando tímidamente, como experiencias exploratorias. En este sentido, en los diversos ámbitos del Gobierno nacional y asociados a gobiernos departamentales y municipales, dos programas podrían catalogarse como portadores de futuro en el aporte de la cultura a la nueva agenda de gobierno. Son programas que no buscan innovar simplemente por buscar lo nuevo, sino por promover la transformación social.

Se trata de las Agendas Municipales de Cultura (AMC), del programa Uruguay Integra de la Dirección de Descentralización e Inversión Pública de la Oficina de Planeamiento y Presupuesto (OPP), y el Sistema de Circulación Cultural (SCC), de la Dirección Nacional de Cultura del Ministerio de Educación y Cultura junto con la Red de Directores de Cultura de las intendencias del país.

Ambas se caracterizan por una concepción integralista; es decir, escapan al común denominador de la mayoría de las políticas de impronta focalizada, que en general tienen un solo punto de mira: o apoyan infraestructura cultural, o promueven la creación o la producción artística, o el rescate y la puesta en valor de las tradiciones, o apoyan procesos formativos, el impacto en las audiencias, etcétera.

Los dos programas referidos aúnan diferentes líneas de acción, tales como formación, mejoramiento de infraestructura, promoción de contenidos culturales, aportes a la financiación, alcance y apropiación ciudadana. Otro aspecto en común es la horizontalidad el tender a entramar redes. El planteo de trabajo tiene un enfoque asociativo y colaborativo, con una lógica participativa, de cooperación y complementariedad.

Las AMC se implementan mediante un diseño participativo en cada municipio o entre municipios asociados. Reciben apoyo para establecer capacidades locales propias mediante acciones de capacitación y se les brinda financiación para desarrollar parcial o totalmente la propuesta de agenda cultural. 
El scC también comienza con la asociación entre la DNC del MEC y las intendencias y los municipios participantes, que confeccionan una programación regional compartida y complementan esfuerzos para solventarla, proyectan un mapeo en la infraestructura cultural dispuesta en cada lugar y relevan necesidades en equipamiento y capacitación técnica, a lo que se integra una línea de formación de públicos y en gestión cultural.

Las experiencias transitadas son en cierto modo piloto y seguramente queden muchos elementos por ajustar. No obstante, en un paisaje neutro que comienza a inquietar a diversos actores sociales, los programas referidos bien pueden ser una muestra de que la cultura es un componente clave en una eventual renovación de la agenda de gobierno de izquierda.

\section{Referencias bibliográficas}

Mendoza, J. J. (5 de enero, 2018). «La disolución del campo cultural: Entrevista con Néstor García Canclini». Clarín, Revista. Recuperado de https:// www.clarin.com/revista-enie/ideas/disolucion-campo-cultural_0_HykCd8TmM.html.

Pascual i Ruiz, J. y Dragojević, S. (2007). «Guía para la participación ciudadana en el desarrollo de políticas culturales locales para ciudades europeas». Bucarest: Fundación Europea de la Cultura, Fundació Interarts y Asociación Ecumest. Recuperado de http://www.ecumest.ro/pdf/Guia_participation_ciudadana_ESP_ web.pdf.

UNESCO (1998). Conferencia intergubernamental sobre políticas culturales para el desarrollo: Informe final. Recuperado de: http://www.lacult.unesco.org/ docc/1998_Conf_Intergub_sobre_pol_cult_para_des.pdf.

UNESCO (2004). Declaración Universal sobre la Diversidad Cultural: Una visión, una plataforma, conceptual, un semillero de ideas, un paradigma nuevo. Lima: UNESCO. (Serie sobre Diversidad Cultural, 1). Recuperado de http://unesdoc.unesco.org/images/0012/001271/127162s.pdf.

UNESCO (2005). Convención sobre la Protección y la Promoción de la Diversidad de las Expresiones Culturales. Recuperado de http://unesdoc.unesco.org/ images/0014/001429/142919s.pdf.

Yang, E.; Abraham, F. (23 de octubre, 2016). «Hay mucha actividad cultural, pero poca producción de arte: Entrevista». La Izquierda Diario. Recuperado de https://www.laizquierdadiario.com/Hay-mucha-actividad-cultural-pero-pocaproduccion-de-arte. 